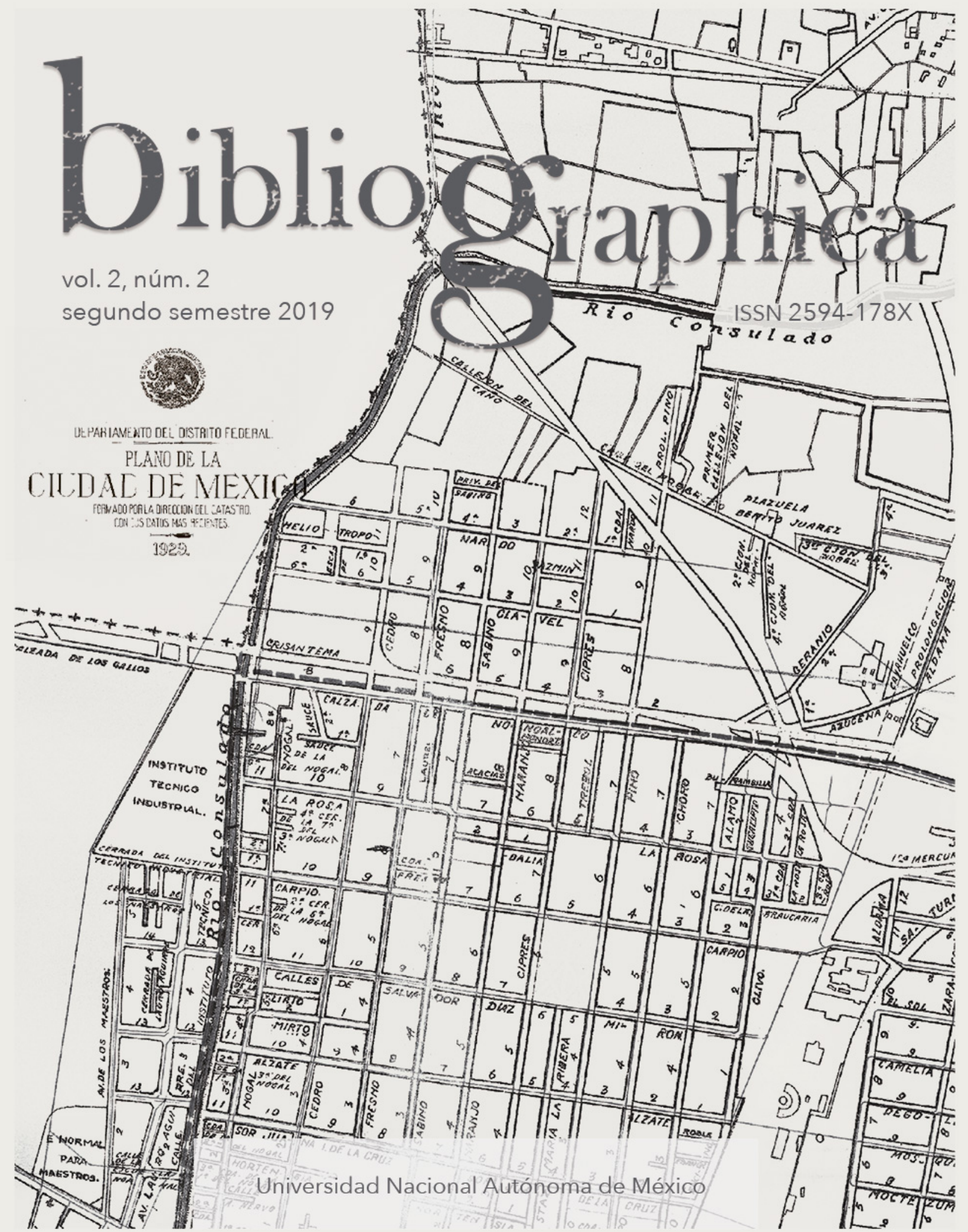




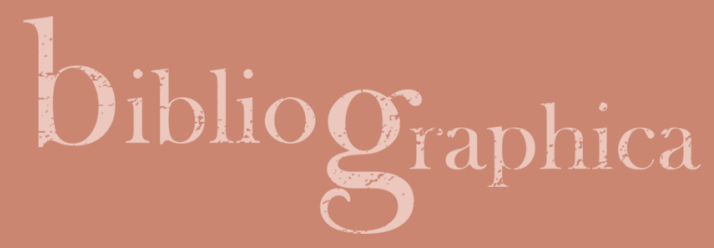

\section{El ISBN y su utilidad para la investigación bibliográfica}

The ISBN and its Utility for Bibliographical Research

Fernando Gruz Quintana

fernandocruzquintana@gmail.com

Universidad Nacional Autónoma de México

Instituto de Investigaciones Bibliográficas

Recepción: 26.04.2019 / Aceptación: 05.06.2019

DOI: https://doi.org/10.22201/iib.bibliographica.2019.2.52 
Resumen

Palabras clave

Abstract

Keywords
Artículo sobre la historia del International Standard Book Number (ISBN), desde que fue creado hasta su expansión por el mundo. Menciona el contexto sociocultural de su surgimiento, enfatizando cómo llegó a América Latina y el Caribe. El primer objetivo de este trabajo es conocer los pormenores de esa historia, y en segundo lugar repensar la utilidad del ISBN en contextos académicos y de investigación del libro. Tras revisar cuáles son los elementos que componen este código estandarizado, se explica cómo podría sacarse provecho de él en los campos de investigación bibliográfica y bibliológica. Finalmente, se reflexiona en torno a cuáles son las problemáticas generales que han impedido que el ISBN pueda consolidarse como una herramienta de trabajo indispensable para bibliotecarios, editores e investigadores.

ISBN; bibliología; bibliografía; industria editorial; América Latina y el Caribe.

This article aproaches the history of the International Standard Book Number (ISBN), from its creation to its expansion around the world. In this paper, the socio-cultural context of its emergence is mentioned, emphasizing how it arrived to Latin America and the Caribbean. The first objective of this work is to know the details of that history, and secondly to rethink its usefulness in scholarly and book research contexts. After reviewing which elements form this standardized code, this paper explains how to benefit from it in bibliographic and book science research environments. Finally, this text reflects on which are the general problems that have prevented the ISBN from consolidating as an essential work tool for librarians, editors and researchers.

ISBN; book science; bibliography; publishing industry; Latin America and the Caribbean. 


\section{Introducción}

En algunos entornos de investigación bibliográfica suele verse con cierta reticencia el estudio del comercio de los libros que ocurre hoy en día. Muchos otros son los principales intereses de estudio bibliográfico, y eso es entendible; probablemente lo más importante sea poder desentrañar, clasificar, explicar e interpretar el contenido de las publicaciones de una nación. No obstante, pensamos que el estudio del libro desde el punto de vista de su producción y comercialización actuales puede contribuir a cumplir de mejor manera todos los propósitos de investigación bibliográfica. Este trabajo ${ }^{1}$ pretende transmitir esa idea al hacer un breve recuento histórico del surgimiento del International Standard Book Number (ISBN) y mostrar el provecho académico y comercial que pudiera obtenerse de él.

Internet y la digitalización actual han propiciado cambios trascendentales en la manera de procesar y obtener información alrededor del mundo, y estas modificaciones, lejos de ser características pasajeras de las sociedades, han derivado en transformaciones profundas en todos los sectores productivos, especialmente en aquellos del ámbito cultural. Aunque, como veremos, el ISBN surgió en un contexto donde su administración no dependía de los recursos digitales y en línea, hoy esto sí ocurre. Precisamente estas características tecnológicas permiten que el aprovechamiento y la lectura de este código con finalidades de investigación sean insospechados.

A pesar de que el ISBN es bien conocido en los entornos bibliotecarios y de investigación del libro, poco se sabe a propósito de su origen y la enorme utilidad que pudiera tener en tareas de generación de conocimiento. La idea de aprovechar la información de códigos como éste es una demanda existente no sólo para el caso del análisis del libro, sino de toda la producción cultural.

es necesario contar con una metodología estándar y periódica que permita dar seguimiento al comportamiento y evolución del valor de las industrias culturales en el tiempo. Aunque como parte de la contabilidad nacional y derivado del esfuerzo de las cámaras reguladoras de algunos subsectores se cuenta con datos independientes de la mayor parte de las ramas que integran a las industrias culturales, lo cierto es que no se tiene una medición de la industria

\footnotetext{
${ }^{1}$ Fernando Cruz Quintana, Programa de Becas Posdoctorales en la UNAM, becario del Instituto de Investigaciones Bibliográficas, asesorado por la doctora Isabel Galina Russell.
} 
cultural mexicana, tal y como se cuantifica cada mes el valor de la industria minera, la de la construcción o el sector automotriz. ${ }^{2}$

La utilización del ISBN con finalidades de investigación pudiera ser igualmente útil para la academia y el sector productivo de libros. Del mismo modo, la información que puede extraerse de sus registros brindaría evidencias sobre el impacto de las políticas gubernamentales en relación con los programas de edición de libros. Además de destacar las virtudes en la utilización de este código estandarizado, en este artículo se mencionarán también algunas de las problemáticas que han entorpecido su gestión, muchas de las cuales dependen de variables sociales propias de los países en donde es tramitado.

\section{La historia del ISBN}

A propósito de la vasta producción editorial a nivel mundial, Gabriel Zaid plantea una hermosa paradoja en su ensayo Los demasiados libros: "Los libros se publican a tal velocidad que nos vuelven incultos. Si uno leyera un libro diario, estaría dejando de leer 4 mil, publicados el mismo día. Es decir: sus libros no leídos aumentarían 4 mil veces más que sus libros leídos. Su incultura, 4 mil veces más que su cultura". ${ }^{3}$ Si consideramos que el autor mexicano escribió estas palabras en 1996, podemos estar seguros de que esas cifras han aumentado exponencialmente. Basta un recorrido por las ferias del libro, o por los anaqueles de las bibliotecas -cada vez más necesitadas de espacio- para cerciorarnos de que efectivamente existen demasiados libros. ${ }^{4}$ En este contexto, en donde las lecturas crecen a tasas más altas que los índices poblacionales, se justifica la existencia de un código estandarizado que permita identificar la unicidad de una obra en un mundo de publicaciones.

El origen del ISBN se remonta a 1965, en un contexto editorial anglosajón. En aquel año la editorial WHSmith - principal minorista de libros en el Reino Unido de aquel entonces-, la Bibliografía Británica Nacional y la Asociación Británica de Editores idearon un sistema de numeración llamado Standard Book Number (SBN). Para la creación de este código dichas organizaciones contra-

\footnotetext{
${ }^{2}$ Lucía Emanuela Martín, "Entorno sectorial del libro e industrias culturales en México", en Informe Omniprom 2010 sobre el libro en México (México: LID Editorial Mexicana, 2011), 33. ${ }^{3}$ Gabriel Zaid, Los demasiados libros (México: Océano, 1996), 20.

${ }^{4}$ Véase Cámara Nacional de la Industria Editorial Mexicana, Indicadores del sector editorial privado en México, 2014 (México: Caniem, 2015).
} 
taron al profesor Gordon Foster del Trinity College Dublin, quien propuso la utilización de una cifra de 9 dígitos que permitiera identificar el editor y el título de una obra. ${ }^{5}$

Aunque en la creación del SBN participaron instancias editoras y bibliotecarias, en gran medida estuvo motivada por la necesidad de contar con registros que facilitaran la gestión comercial de las obras publicadas por las editoriales de aquel país. Tal fue el éxito de esta propuesta que el SBN se utilizó inmediatamente en Estados Unidos para fines comerciales, y en Canadá, Australia, Dinamarca, Suecia y Holanda en el ámbito bibliográfico. ${ }^{6}$ En tan sólo cinco años de existencia el SBN logró consolidarse como un excelente mecanismo para la normalización bibliográfica, y en 1970 fue aceptado por la Organización Internacional de Normalización (ISO por sus siglas en inglés). ${ }^{7}$

La ISO fue fundada el 23 de febrero de $1947^{\circ}$ y una de sus finalidades era crear estándares internacionales que permitieran normalizar la información e identificar la generación de datos sobre diferentes producciones humanas. Desde entonces hasta la fecha se han creado aproximadamente 20 mil estándares que abarcan lo mismo productos manufacturados y tecnología, que seguridad alimenticia, agricultura y sanidad. Por lo anterior es que la incorporación del ISBN como uno más de sus estándares estuvo justificada.

Una vez que el ISBN fue reconocido por la ISO, la historia de este número estandarizado continuó con la creación de las distintas agencias nacionales de ISBN en el mundo; de 1970 a la fecha se han sumado cada vez más, a tal grado que hoy existen alrededor de 150 en todo el mundo. ${ }^{9}$ Si bien es cierto que durante el otorgamiento del código no debieran existir confusiones o duplicados, se presentan algunas problemáticas propias de cada uno de los diferentes países donde el código es asignado, y éstas pueden entorpecer el propósito de respetar la unicidad de las publicaciones.

\footnotetext{
${ }^{5}$ Centro Regional para el Fomento del Libro en América Latina y el Caribe (Cerlalc), Panorama de las agencias del ISBN en Iberoamérica. Diagnóstico y recomendaciones para su fortalecimiento (Colombia: Cerlalc, 2018), 15.

6/bid., 16.

7 Renée Register y Thad Mcllroy, The Metadata Handbook. A Book Publishers Guide to Creating and Distributing Metadata for Print and Ebooks ([Estados Unidos]: DataCurate, 2015), 59.

8 Organización Internacional de Normalización (ISO). "All about ISO", acceso el 7 de mayo de 2019, https://www.iso.org/about-us.html.

${ }^{9}$ Agencia Internacional de ISBN, "Las agencias nacionales de ISBN", acceso el 7 de mayo de 2019, https://www.isbn-international.org/es/content/las-agencias-nacionales-del-isbn.
} 
Aquellos eran otros tiempos y la información no fluía a ritmos tan vertiginosos como hoy en día. Pasarían unos años antes de que el ISBN fuera utilizado en México, sin embargo, su enorme utilidad se discutía ya en los ámbitos de estudio del libro:

El fin es contar con un método eficaz y económico de comunicación entre las casas editoras y las casas vendedoras de libros que permita sistemas de distribución más rápidos y eficientes; todo editor, distribuidor, librero, bibliotecario estarían, así, en condiciones de trabajar más eficientemente en cuanto a pedidos por escrito, procesamientos y préstamos interbibliotecarios, tareas que hasta ahora, debido a la falta de oportunidad, se traducen en pérdida de tiempo. Una vez completada la numeración normalizada internacional del libro se dispondrá de un medio rápido y sencillo para la localización, recuperación y divulgación de los datos bibliográficos. ${ }^{10}$

Las palabras de Gloria Escamilla resultan reveladoras de los empeños del comercio internacional por estandarizar la información de los libros, sin embargo, como ella misma admite, otros actores involucrados en la vida de las publicaciones podrían sacar un enorme provecho de la implementación de este código. En el contexto iberoamericano del libro no fue una casualidad que las primeras cuatro naciones que utilizaron el ISBN son las que tienen un sector editorial más fuerte: España abrió su agencia en 1972, México lo hizo en 1977, Brasil en 1978 y Argentina en 1982. ${ }^{11}$

Justo en el contexto de los años 80 y hasta finales del siglo XX, el Centro Regional para el Fomento del Libro en América Latina y el Caribe (Cerlalc) ${ }^{12}$ jugó un rol importante para promover su implementación entre los países integrantes. Además de lo anterior, en el año 2004 esta organización, con la Cámara

\footnotetext{
${ }^{10}$ Gloria Escamilla, "El ISBN y el ISSN", Boletín del Instituto de Investigaciones Bibliográficas (enero-diciembre de 1974): 357.

${ }^{11}$ Cerlalc, Panorama de las agencias del ISBN en Iberoamérica, 33.

12 El Cerlalc fue creado en 1971 por medio de un Acuerdo de Cooperación Internacional entre Colombia y la UNESCO, al cual se han adherido Argentina, Bolivia, Brasil, Chile, Costa Rica, Cuba, Ecuador, El Salvador, España, Guatemala, Honduras, México, Nicaragua, Panamá, Paraguay, Perú, Portugal, República Dominicana, Uruguay y Venezuela. Una de sus principales funciones es fomentar la investigación, divulgación y construcción del conocimiento, en materia del libro y la lectura. Su existencia se justifica porque generan aportaciones que contribuyen a la implementación de los objetivos del programa estratégico de la UNESCO para beneficio de los países miembros.
} 
Colombiana del Libro, diseñó un software especializado para la solicitud de registros de ISBN en línea, denominado RISBN, que sería utilizado posteriormente por todos sus países miembros.

México fue el primer país de América Latina que comenzó a gestionar el registro de ISBN, actualmente esta labor se lleva a cabo mediante el Instituto Nacional de Derechos de Autor (Indautor), ${ }_{13}^{13}$ situación que resulta excepcional cuando se compara con el caso de otras naciones de la región: el caso mexicano es el único en donde la asignación del ISBN depende directamente de una oficina responsable de salvaguardar los derechos autorales de los creadores de la nación. Del total de los países iberoamericanos, en $62 \%$ de ellos el trámite de este código estandarizado es responsabilidad de instituciones públicas y en $32 \%$ de privadas. ${ }^{14}$ En el primer caso, es casi siempre la Biblioteca Nacional de las diferentes naciones la encargada de hacer este procedimiento; ${ }^{15}$ y en el caso de las segundas, es por medio de las asociaciones gremiales o cámaras del libro.

Esta información no es banal y revela la concepción imperante (ya sea patrimonial o comercial) que se tiene sobre los libros. Piénsese por ejemplo en la utilidad que el registro de ISBN pudiera tener para el cumplimiento del decreto de Depósito Legal. ¿Qué pasaría si la Biblioteca Nacional de un país supiera de antemano cuál es la totalidad de ejemplares que se han editado en un año? Con esa información podría generarse una base de datos histórica que facilitaría el control del patrimonio escrito de una nación. Es conveniente preguntarse sobre la posibilidad de que esos datos puedieran incluso ser de conocimiento público, tal y como ocurre con Colombia, en donde a través de las páginas de Internet de la Cámara Colombiana del Libro y la Biblioteca Nacional de Colombia, puede hacerse un seguimiento de los registros de ISBN y del Depósito Legal de aquel país. ${ }^{16}$

${ }^{13}$ El Indautor fue creado en 1997 y sustituyó a la Dirección General de Derecho de Autor de la Secretaría de Educación Pública (SEP), que desde 1977 gestionaba la asignación del ISBN en México. En 2015 Indautor se convirtió en un órgano desconcentrado de la Secretaría de Cultura de México y aunque cuenta con una autonomía orgánica, el hecho de que dependa presupuestalmente de esta secretaría limita muchas de las actividades que pudieran realizarse en materia no sólo de libros, sino también de publicaciones periódicas y de derechos de autor en general.

${ }^{14}$ Cerlalc, Panorama de las agencias del ISBN en Iberoamérica, 59.

${ }^{15}$ Con excepción de Venezuela y Cuba, en donde la secretaría o ministerio de cultura a través de alguna de sus oficinas lleva a cabo esta tarea, y México, en donde-como ya se dijo- se tramita a través del Instituto Nacional de Derechos de Autor (Indautor).

${ }^{16}$ Cámara Colombiana del Libro, "Base de datos de ISBN de Colombia", acceso el 7 de mayo de 2019, https://isbn.camlibro.com.co/catalogo.php. Biblioteca Nacional de Co- 
A muy grandes rasgos, esa ha sido la historia de los derroteros del ISBN desde que llegó de Europa hasta América. Es importante recordar la función originaria de este código estandarizado: identificar la unicidad de las publicaciones no periódicas. En la historia de poco más de medio siglo de existencia, el ISBN ha tenido que adaptarse a las necesidades propias de los momentos históricos de la producción de la cultura en la segunda mitad del siglo pasado, de modo tal que diversos objetos diferentes de un libro impreso se han añadido como bienes que pueden ser normalizados mediante este registro. Aunque en principio sólo eran consideradas las publicaciones monográficas basadas en texto y puestas a disposición del público a través de una obra impresa, en la actualidad éstas son todas las producciones que pueden obtener un ISBN: ${ }^{17}$

- Libros y folletos impresos.

- Capítulos individuales o secciones de una publicación, si se distribuyen separadamente.

- Publicaciones en braille.

- Mapas.

- Películas, vídeos y diapositivas educativas o formativas.

- Audiolibros en casete, CD o DVD (libros hablados).

- Publicaciones electrónicas tanto en un soporte físico (cintas magnéticas, disquetes o CD-ROM) como en Internet.

- Copias digitalizadas de publicaciones monográficas impresas.

- Publicaciones en microformato.

- Software educativo o formativo.

- Publicaciones multimedia (siempre que el elemento principal esté basado en texto).

- Publicaciones bajo demanda (que es un método para imprimir tirajes cortos).

lombia, "Depósito Legal", acceso el 7 de mayo de 2019, http://bibliotecanacional.gov.co/ es-co/servicios/profesionales-del-libro/deposito-legal.

${ }^{17}$ La siguiente lista se tomó directamente del sitio de Internet de la Agencia Internacional de ISBN y es importante señalar que, pese a su aparente vaguedad, considera los libros electrónicos, que entrarían bajo el rubro de Publicaciones electrónicas con un soporte en Internet. También es imperativo entender que no cualquier CD o película puede obtener un registro; en el primer caso se trata de discos compactos que sólo contengan audio hablado o instructivo, y las películas también deben ser didácticas o tener propósitos educativos, nunca con contenido de entretenimiento. Agencia Internacional de ISBN, "Alcance y asignación de ISBN", acceso el 7 de mayo de 2019, https://www.isbn-international.org/ es/content/alcance-y-asignaci\%C3\%B3n-de-los-isbn. 
Además de la incorporación de todas estas producciones -como productos susceptibles de ser normalizados por el ISBN-, la propia edición de libros tuvo un crecimiento exponencial hacia finales del siglo XX. ${ }^{18}$ Con el surgimiento de los libros digitales se consideró la posibilidad de crear nuevos estándares que normalizaran esos nuevos formatos, pero la idea no prosperó: "In the early 2000s, there was considerable debate about whether digital publications in the supply chain should use a numbering system completely different from ISBNs. After investigating some options, this was felt to be complex and restrictive, as well as unnecessary, since the scope of ISBN encompasses books in any possible format". ${ }^{19}$

Estas dos situaciones mencionadas, más la idea de que el ISBN fuera compatible con el sistema de identificadores únicos para el comercio internacional, favorecieron que el número se ampliara de 10 a 13 dígitos en el año 2007, que es tal y como se mantiene hasta la fecha. En suma, conviene recordar que aunque este artículo menciona las posibles utilidades que el ISBN pudiera tener en los entornos bibliotecarios y de investigación académica del libro, la razón de ser de este código es eminentemente de carácter comercial y global. Pese a esto último, el ISBN puede ser de interés para editores, libreros, bibliotecarios e investigadores que tratan con el análisis de la producción editorial de una nación.

\section{El ISBN en entornos académicos y de investigación}

En ámbitos académicos y comerciales existen concepciones diferentes sobre aquello que constituye un libro, sin embargo, estas ideas en ocasiones parecieran ser excluyentes, cuando no necesariamente lo son. Por un lado, la investigación bibliográfica no suele prestarle tanta importancia a los aspectos productivos y comerciales del libro, como si hablar de ellos fuera en detrimento de la grandeza de las obras; a la inversa, en los entornos comerciales existe un recelo por compartir cualquier tipo de información con investigadores externos, y mu-

\footnotetext{
${ }^{18}$ Fernando Escalante Gonzalbo, A la sombra de los libros. Lectura, mercado y vida pública (México: El Colegio de México, 2007), 256.

19 "A principios de la década de 2000, hubo un debate considerable sobre si las publicaciones digitales en la cadena de suministro deberían usar un sistema de numeración completamente diferente de los ISBN. Después de investigar algunas opciones, se consideró que esto era complejo y restrictivo, además de innecesario, ya que el alcance del ISBN abarca los libros en cualquier formato posible". Stella Griffiths, "ISBN: A History", Information Standards Quarterly 27, núms. 2-3 (verano y otoño de 2015): 47. La traducción es mía.
} 
chas veces se desconoce que la generación de conocimiento sobre el mundo de la edición también puede serles de utilidad. En la búsqueda de consensos y trabajo colaborativo, los registros de ISBN podrían ser un baluarte que apoye de igual manera a los sectores editoriales y de investigación.

Hoy en día cada uno de los registros de ISBN se compone de 13 dígitos agrupados en 5 elementos, son series de números separados entre sí mediante un espacio o un guión. Cada una de esas agrupaciones de cifras permite dar cuenta de cosas diferentes:

1. Prefijo 978 y 979 . Estos números nunca cambian y son asignados de acuerdo con el estándar "Número de Artículo Internacional" que se utiliza para normalizar procedimientos comerciales a nivel mundial.

2. Grupo de registro. Identifica a un determinado país, una región geográfica o un área lingüística que tenga agencia de ISBN.

3. Titular. Corresponde a un determinado editor o sello editorial.

4. Publicación. Identifica una determinada edición y formato.

5. Dígito de control. Es un grupo de números verificador que valida matemáticamente al resto del número.
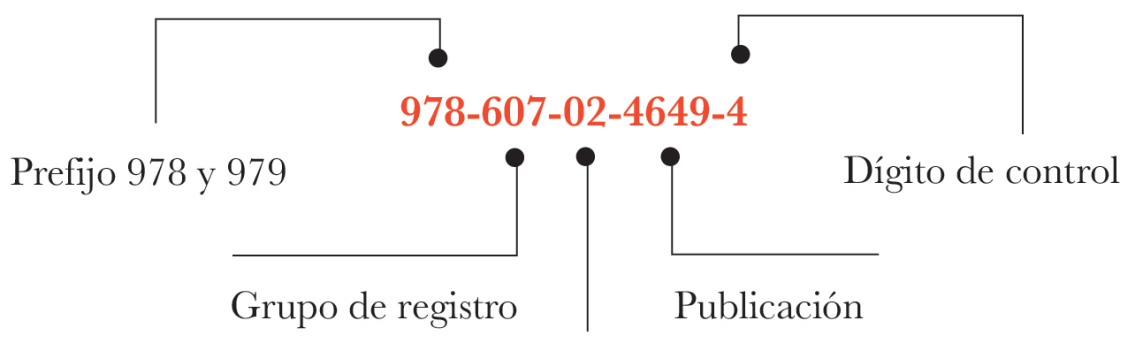

Titular

No existe arbitrariedad en la asignación de los registros de ISBN ni se trata solamente de una cifra ascendente hasta el infinito. Como podemos observar, cada grupo de elementos tiene asociada una categoría en específico y al hacer una revisión de ellos podríamos tener lecturas globales de cómo se comporta la publicación de libros por región geográfica de acuerdo, por ejemplo, al titular de la edición o el tipo de formato. Este primer análisis del código es por demás provechoso, pero no es el único que puede hacerse. 
Aunque los elementos contenidos en los cinco grupos de números de un registro de ISBN son útiles, realmente el gran provecho de investigación que puede dársele a este código tiene que ver con la manera en que es solicitado. Como se mencionó, los países de América Latina y el Caribe miembros del Cerlalc utilizan el software RISBN que creó este centro para tramitar el ISBN. El procedimiento se lleva a cabo en línea y para finalizarlo hay que llenar una serie de metadatos ${ }^{20}$ que caracterizan a la obra para la cual se solicita un ISBN. Los campos requeridos son los siguientes: ${ }^{21}$

a. Información del editor. Por medio de ella puede identificarse a la empresa editorial o persona responsable de la edición.

b. Datos de la ubicación. Dirección, teléfono, página de Internet, ciudad y correo electrónico de la empresa editorial o persona responsable de la edición.

c. Datos de caracterización. Actividad principal de la editorial (servicios editoriales, de distribución...) y la naturaleza jurídica de la misma.

d. Fecha y el lugar de solicitud.

e. Tipo de obra. Con lo cual se puede saber si las publicaciones son independientes o si forman parte de una colección más grande.

f. Título, subtítulo y sello editorial. Aquí se registra el nombre de la obra y se especifica el sello al que pertenece.

g. Clasificación temática y subtemática. De acuerdo con el sistema de clasificación Dewey, se eligen los aspectos que mejor describen el contenido del libro.

h. Autores y colaboradores. Nombre de quienes participaron en la publicación, aclarando el tipo de colaboración de cada uno de ellos.

i. Información sobre la traducción. Para saber el idioma original en el que estaba escrita una obra y el idioma en el cual se publica una traducción, así como el nombre de los responsables de este tipo de trabajo.

\footnotetext{
${ }^{20}$ Los metadatos son grupos de información que describen al objeto o recurso y pueden ser utilizados como etiquetas para clasificar contenidos digitales. Estos elementos son indispensables en el trabajo de recuperación de información en las bases de datos y son también el pilar de lo que hoy se conoce como "web semántica".

${ }^{21}$ Instituto Nacional de Derechos de Autor (Indautor), Guía de llenado del sistema RISBN 5.1 (México: Secretaría de Cultura, 2019), acceso el 13 de mayo de 2019, https://indautor. gob.mx/isbn/llenar.html.
} 
j. Información sobre la edición. Con esta información puede conocerse el número de edición, saber si se trata de una coedición y quiénes participan en ella, así como la ciudad de edición.

k. Datos de comercialización. En el caso de los impresos, se coloca información sobre el tiraje. También hay que mencionar si la publicación es o no comercializable y su precio de venta al público.

I. Descripción física. Para distinguir la especificidad de la obra registrada en función de si es o no un libro impreso, electrónico (y en qué formato se encuentra), en braille, etcétera. Los tipos de encuadernación e impresión, y el tipo de papel utilizado.

De acuerdo con los intereses bibliográficos o bibliológicos de estudio que se tengan, ${ }^{22}$ los datos mencionados en las diferentes categorías solicitadas para tramitar un ISBN pueden ser de mayor o menor utilidad. Uno de los principales retos para sacar provecho de estos registros es conocer las vías para acceder a este tipo de información, y ello depende de la instancia que se encargue de llevar a cabo esa tarea. Como se mencionó, las agencias de ISBN nacionales son administradas por entidades públicas y privadas, lo cual es determinante en el caso de cómo obtener dicha información.

La utilidad del ISBN para la conformación de una bibliografía nacional es insospechada, pero podría ocurrir en distintos niveles sucesivos: por ejemplo, proporcionar los datos estadísticos de la producción editorial de un país y con ello brindar evidencias sobre el impacto de políticas gubernamentales en relación con programas educativos, lingüísticos, económicos, etcétera. ${ }^{23}$ Piénsese en la precisión de la información que se puede generar: alguien podría obtener el registro histórico de ISBN de una editorial (¿por qué no de una pública, como el Fondo de Cultura Económica?) y hacer selecciones de información que llevaran a determinar los porcentajes temáticos, así como el valor promedio de esas ediciones.

${ }^{22}$ Y también en otros contextos de investigación, como muestra el siguiente ejemplo: Elena Guardiola y Javier Sánchez-Carbonell, "Las bases de datos de ISBN como instrumento para la recuperación y análisis de libros sobre un tema específico. Estudio de caso concreto: el alcoholismo", Revista Española de Documentación Científica 19, núm. 3 (1996): 292-303.

${ }^{23}$ Maja Žumer, ed., Bibliografías nacionales en la era digital: Guía y nuevas orientaciones ([España]: Grupo de Trabajo de la IFLA sobre Directrices para Bibliografías Nacionales, 2009), 19, acceso el 13 de mayo de 2019, https://www.ifla.org/files/assets/bibliography/ nb_spanish_version.pdf. 
Otra idea (también con una finalidad de carácter público) podría ser la de realizar un registro histórico de cuáles son las temáticas que más se editan en determinada región geográfica, y a partir de él formular preguntas de investigación. ¿Por qué en este sitio se editan muchos contenidos de estas características? ¿Es producto de ciertas condiciones particulares de edición o más bien depende del consumo de determinados contenidos que se elaboran en esa región?

A nivel macro, como puede intuirse, el estudio de los registros de ISBN ayudaría a plantear líneas de acción, y también en la solución de ciertas incógnitas. Con preguntas como las anteriores conviene reflexionar sobre las funciones de las instancias que asignan y gestionan IOS ISBN: ¿no deberían ser también las responsables de publicar información que describa el comportamiento del sector editorial de un país?

Los registros de ISBN podrían ser útiles incluso para la administración pública de la cultura. Los gobiernos en turno pueden tener elementos para conocer de manera pormenorizada el estado de la edición en un país. Con base en información como ésta, se alcanza una mejor posición para dictar políticas públicas encaminadas a fortalecer la producción, el comercio y la lectura de los libros. No es un hecho fortuito que en muchos de los países de América Latina y en el mundo, la asignación del ISBN se lleve a cabo desde alguna dependencia estatal.

En comparación con la antigua historia del libro, la idea de conformar una "bibliografía nacional" es mucho más reciente. La preocupación por documentar la producción libresca de un país surgió apenas a mediados del siglo XIX, ${ }^{24}$ pero hoy en día tenemos una herramienta muy útil para continuar con ese trabajo en los años por venir. Es cierto que el ISBN tiene la limitante de no considerar la producción editorial previa a su existencia, y que habría muchas complicaciones en utilizarlo para evaluar el trabajo editorial de los años 70,80 y 90 del siglo pasado. Pese a ello, el tiempo presente y futuro tiene condiciones inigualables para dejar registros fehacientes que documenten el desarrollo de los entornos del libro en las diferentes naciones.

\footnotetext{
${ }^{24}$ Véase Mona Madsen, "The National Bibliography in the Future: New Recommendations", Alexandria. The Journal of National and International Library and Information Issues 12, núm. 1 (2000): 45-50.
} 


\section{Conclusiones}

Si bien es cierto que los registros de ISBN tienen un costo y son realmente útiles desde el punto de vista comercial, es pertinente preguntarse sobre el tipo de información que contienen y, sobre todo, si esos datos deberían ser de acceso público. En tanto que son instancias de distinto origen las que llevan a cabo este trámite en todos los países, la pregunta debe realizarse desde cada nación en particular.

En el caso de México, que el Indautor sea la institución responsable de esta tarea y además se financie con recursos públicos es un argumento a favor para dar acceso abierto a su información. Es cierto que existen ciertos datos personales que pudieran reservarse mediante las diferentes legislaciones de protección de datos personales vigentes en el país, pero otros más pueden dar mucha luz para comprender qué tipo de publicaciones se editan anualmente.

Aunque el trámite de registro y las bases de datos históricas del ISBN estén en manos de Indautor, sería ideal que tanto la Cámara Nacional de la Industria Editorial Mexicana (Caniem) ${ }^{25}$ como la Biblioteca Nacional de México (BNM) ${ }^{26}$ pudieran contar con un respaldo de esa información. La Caniem, por ejemplo, podría generar información que tratara -con lujo de detalle y a partir de la medición de diferentes variables- sobre el estado de desarrollo que tiene la industria, mientras que a la BNM le brindaría la oportunidad de tener un elemento para cotejar el estado del Depósito Legal que hacen los editores. Con los datos también podría elaborarse una bibliografía nacional (de acceso público) que

${ }^{25}$ La Cámara Nacional de la Industria Editorial Mexicana (Caniem) fue fundada el 23 de marzo de 1964 con el objetivo de ser la institución responsable de representar los intereses generales de la industria editorial y los particulares de sus afiliados, de acuerdo con lo dispuesto por la Ley de Cámaras Empresariales y sus Confederaciones. Desde aquel año y hasta la fecha, sus afiliados han sido personas físcas o morales dedicadas exclusiva o preponderantemente a la edición de libros y publicaciones periódicas publicados por cualquier sistema de impresión o mediante alguna plataforma electrónica.

${ }^{26}$ La Biblioteca Nacional de México (BNM) es una institución centenaria creada en 1867 por decreto del entonces presidente de México, Benito Juárez. En aquel año sus instalaciones estaban en el antiguo Templo de San Agustín. En 1914 la administración de la BNM dejó de depender de la Secretaría de Educación Pública y se vinculó a la Universidad Nacional de México (institución que alcanzó su autonomía en 1929). En 1967 se fundó el Instituto de Investigaciones Bibliográficas, que entre otras funciones tiene la de administrar la BNM, así como estudiar los fondos bibliográficos que contiene y generar una bibliografía nacional. Finalmente, en 1979 la BNM se trasladó de San Agustín al recinto que actualmente la alberga en la Zona Cultural de Ciudad Universitaria. 
tuviera, al menos, los diferentes filtros de información que el trámite de ISBN ya demanda en la actualidad.

Es importante considerar que los elementos que componen al ISBN-como los de cualquier estándar que normaliza las producciones culturales- no son inmutables, más bien son perfectibles y necesitan una constante adecuación para dar cuenta de los nuevos tipos de productos editoriales que pudieran existir. Como se dijo, en América Latina y el Caribe esa tarea de normalizar los registros la lleva a cabo el Cerlalc, que desempeña el rol de máximo representante ante la Agencia Internacional de ISBN, y actualmente están implementando en los diferentes países de la región un nuevo software para la asignación de ISBN. Esta nueva herramienta ya funciona en naciones como Colombia y sus resultados pueden apreciase a través de la página en línea de la Cámara Colombiana del Libro. ${ }^{27}$

Sin importar cuál sea el software utilizado para llenar la información en las solicitudes de ISBN, uno de los mayores problemas que hay en su gestión es el perfil diferente de las personas que realizan esta actividad. Aunque hay ciertos rubros de información básica que cualquier individuo con mínimos conocimientos sobre el tema pudiera llenar, existen otros muy técnicos o especializados que deben capturarse con mucho cuidado. Por razones como ésta se vuelve necesario que las bases de datos de los registros ISBN pudieran tener revisiones periódicas encaminadas a detectar este tipo de problemas, y gracias a ellas poder corregir cualquier error.

En un mundo con grandes prodigios técnicos en materia de comunicación como el que vivimos hoy en día, los registros del ISBN deberían estar interconectados con las bibliotecas nacionales para que la cosecha de información pudiera darse de manera automatizada (sin que eso implique la ausencia de gestión humana). Si bien los ISBN se tramitan por país, existen autoridades regionales y una internacional en la materia. ¿Por qué no hablar del registro público mundial de ISBN? Aunque la pretensión inicial sería normalizar los registros nacionales de ISBN (lo cual podría hacerse si todas las bibliotecas nacionales tuvieran acceso a las bases de datos respectivas), podríamos pensar en la creación de una verdadera bibliografía internacional o mundial.

Pensando en una propuesta concreta que conjuntara los esfuerzos de distintas naciones en pro de la utilización del ISBN con finalidades de investigación, en las secciones regionales de la Federación Internacional de Asociaciones de

${ }^{27}$ El "Catálogo de publicaciones" de la Cámara Colombiana del Libro puede consultarse en el siguiente enlace: https://isbn.camlibro.com.co/catalogo.php. 
Bibliotecarios (IFLA por sus siglas en inglés) ${ }^{28}$ se podrían crear propuestas estandarizadas para el manejo de las bases de datos de ISBN. No sería necesario que las bibliotecas nacionales fueran las encargadas de asignar este número estandarizado, labor que podría depender de otras instituciones, pero sí sería fundamental, a riesgo de ser redundante, que la información de los registros de ISBN tenga una utilización más provechosa en un entorno de estudio del libro.

Por último, resta decir que existen muchos otros códigos estandarizados para normalizar las diferentes producciones culturales del hombre. Uno de los más importantes en materia de preservación del patrimonio de una nación es el International Standard Serial Number (ISSN), que surgió poco después del registro para libros y tuvo un desarrollo semejante, sólo que es utilizado para identificar publicaciones seriadas o periódicas, ya sea impresas o en formatos digitales. Si bien muchas de las reflexiones de este trabajo sobre el caso del ISBN podrían aplicar también para el ISSN, su complejidad es tal que debería abordarse por separado.

El tema de la estandarización de la información encuentra hoy un panorama contradictorio: por un lado existen muchas tecnologías que facilitan el procesamiento de la información y la creación de bases de datos, pero por otro hay también una nueva complejidad para atender las producciones digitales, que tienen formas muy específicas y difíciles de controlar. Algunos otros identificadores han tratado de normalizar esta situación, como es el caso del DOI, usado para identificar de forma única cualquier documento electrónico (artículo, libro, revista, mapa, imagen, base de datos, etcétera) y asignarle un enlace permanente. ${ }^{29}$ La utilización de recursos como el DOI no excluye al ISBN o al ISSN, por el

${ }^{28}$ Esta organización mundial fue creada en septiembre de 1927 y tiene como propósito representar los intereses de los servicios de biblioteca e información, al igual que los de sus usuarios. La IFLA organiza encuentros internacionales en donde bibliotecarios de todo el mundo conviven e intercambian experiencias para promover la cooperación e investigación en todos los campos de la actividad bibliotecaria y bibliotecológica. Tomando en cuenta que esta federación cuenta con oficinas regionales en África, Asia y Oceanía, América Latina y el Caribe, tal situación favorecería el trabajo regional y conjunto en la creación de estándares de trabajo con las bases de datos de ISBN.

${ }^{29}$ El Identificador de Objeto Digital (DOI por sus siglas en inglés) es un código alfanumérico que identifica la ubicación de un contenido digital y se expresa a manera de dirección específica asignada o URL (Localizador Uniforme de Recursos). La utilidad de este recurso ha crecido exponencialmente en el ámbito académico: mediante el mismo se puede visibilizar y vincular de manera muy sencilla la producción académica de las instituciones o investigadores. 
contrario, pueden complementarse y hacer que la función de cada uno de ellos se cumpla a cabalidad. La investigación académica vive hoy en día un momento de cambio en el cual las virtudes de la información digital, leída a través de los metadatos de los objetos digitales, nos llevarán a una nueva etapa en la generación del conocimiento; esto, claro, expresado sin ningún matiz de determinismo tecnológico.

\section{Referencias}

Agencia Internacional de ISBN. "Las agencias nacionales de ISBN". Acceso el 7 de mayo de 2019. https://www.isbn-international.org/es/content/las-agen cias-nacionales-del-isbn.

Agencia Internacional de ISBN. "Alcance y asignación de ISBN". Acceso el 7 de mayo de 2019. https://www.isbn-international.org/es/content/alcance-yasignaci\%C3\%B3n-de-los-isbn.

Biblioteca Nacional de Colombia. "Depósito Legal". Acceso el 7 de mayo de 2019. http://bibliotecanacional.gov.co/es-co/servicios/profesionales-del-libro/ deposito-legal.

Cámara Colombiana del Libro. "Base de datos de ISBN de Colombia" y "Catálogo de publicaciones". Acceso el 7 de mayo de 2019. https://isbn.camlibro. com.co/catalogo.php.

Cámara Nacional de la Industria Editorial Mexicana (Caniem). Indicadores del sector editorial privado en México, 2014. México: Caniem, 2015.

Centro Regional para el Fomento del Libro en América Latina y el Caribe (Cerlalc). Panorama de las agencias de ISBN de Iberoamérica. Diagnóstico y recomendaciones para su fortalecimiento. Colombia: Cerlalc, 2018.

Escalante Gonzalbo, Fernando. A la sombra de los libros. Lectura, mercado y vida pública. México: El Colegio de México, 2007.

Escamilla, Gloria. "El ISBN y el ISSN". Boletín del Instituto de Investigaciones Bibliográficas (enero-diciembre de 1974): 357-382.

Griffiths, Stella. "ISBN: A History". Information Standards Quarterly 27, núms. 2-3 (verano y otoño de 2015): 45- 48.

Guardiola, Elena y Javier Sánchez-Carbonell. "Las bases de datos de ISBN como instrumento para la recuperación y análisis de libros sobre un tema específico. Estudio de caso concreto: el alcoholismo". Revista Española de Documentación Científica 19, núm. 3 (1996): 292-303. 
Instituto Nacional de Derechos de Autor (Indautor). Guía de llenado del sistema RISBN 5.1. México: Secretaría de Cultura, 2019. Acceso el 13 de mayo de 2019. https://indautor.gob.mx/isbn/llenar.html.

ISBN Users' Manual. Londres: International ISBN Agency, 2012.

Madsen, Mona. "The National Bibliography in the Future: New Recommendations". Alexandria. The Journal of National and International Library and Information Issues 12, núm. 1 (2000): 45-50.

Martín, Lucía Emanuela. "Entorno sectorial del libro e industrias culturales en México". En Informe Omniprom 2010 sobre el libro en México, 31-55. México: LID Editorial Mexicana, 2011.

Miramontes Vidal, Gabriela Betsabé. "El libro de artista contemporáneo y su relación con el ISBN y el Depósito Legal". Bibliographica 1, núm. 1 (primer semestre de 2018): 181-197. Acceso el 13 de mayo de 2019. https://bibliographica.iib.unam.mx/index.php/RB/article/view/11.

Organización Internacional de Normalización (ISO). "All about ISO". Acceso el 7 de mayo de 2019. https://www.iso.org/about-us.html.

Register, Renée y Thad Mcllroy. The Metadata Handbook. A Book Publishers Guide to Creating and Distributing Metadata for Print and Ebooks. [Estados Unidos]: DataCurate, 2015.

Tat Keh, Hean. "Evolution of the Book Publishing Industry. Structural Changes and Strategic Implications". Journal of Management History 4, núm. 2 (1998): 104-123.

Zaid, Gabriel. Los demasiados libros. México: Océano, 1996.

Žumer, Maja, ed. Bibliografías nacionales en la era digital: Guía y nuevas orientaciones. [España]: Grupo de Trabajo de la IFLA sobre Directrices para Bibliografías Nacionales, 2009. Acceso 13 de mayo de 2019. https://www.ifla. org/files/assets/bibliography/nb_spanish_version.pdf. «b 